\title{
SOLAR FLARES: HIGH-ENERGY RADIATION AND PARTICLES
}

\author{
ERICH RIEGER
}

Max-Planck-Institut für Physik und Astrophysik, Institut für extraterrestrische Physik, 8046 Garching, F.R.G.

\begin{abstract}
Due to the Sun's proximity flares can be investigated in the gamma-ray regime and flare generated particles can be measured in space and related to particular events. In this review paper we focus on the problem of particle acceleration by using as observational ingredients: the fluxes and spectra of particles inferred from gamma-ray measurements and observed in interplanetary space, the temporal characteristics of flares at high-energy X-and gamma-rays and the distribution of gamma-ray flares over the solar disc.
\end{abstract}

\section{Introduction}

The storage and sudden explosive release of energy in sheared magnetic fields involving the acceleration of particles is a common phenomenon occurring in plasmas throughout the Universe from a place as close as the Earth's magnetosphere to objects at cosmological distances such as quasars. The physical understanding of these processes is of basic importance to astrophysics and solar terrestrial physics. Over this vast distance scale the Sun is of crucial importance. Due to its proximity, flares can be investigated in the gamma-ray regime and flare-generated particles can be measured in space and related to particular events, which provides unique information about the acceleration of particles. Furthermore, flares can be localized on the disc, allowing us to study them under different aspect angles. Observations of center-to-limb variations of properties of the high-energy radiation place constraints on flare models.

We begin this article with a brief summary of the mechanisms which produce gammarays and neutrons and then comment on a correlation between the continuum and line radiation during solar flares. We follow with a comparison of particle fluxes measured in interplanetary space and particle number inferred from the gamma-ray measurements. After a discussion of the energy spectra of the primary accelerated particles and of the temporal characteristics of gamma-ray flares relevant particle acceleration mechanisms will be briefly reviewed. Finally, the implications on the particle beaming deduced from the nonuniform distribution of gamma-ray flares on the solar disc will be discussed.

Solar elemental abundance determinations, the anomalous ${ }^{3} \mathrm{He}$ content during certain flares and periodicity studies carried out with energetic events are not covered in this paper. The reader is referred to reviews by Chupp (1984, 1987), Ramaty and Murphy (1987), and Kocharov (1987). 


\section{Production of Gamma-Rays and Neutrons}

The gamma-ray and neutron production mechanisms, most relevant for the Sun, are shown in Figure 1. They can be classified according to the species of the parent particle (electron or proton).

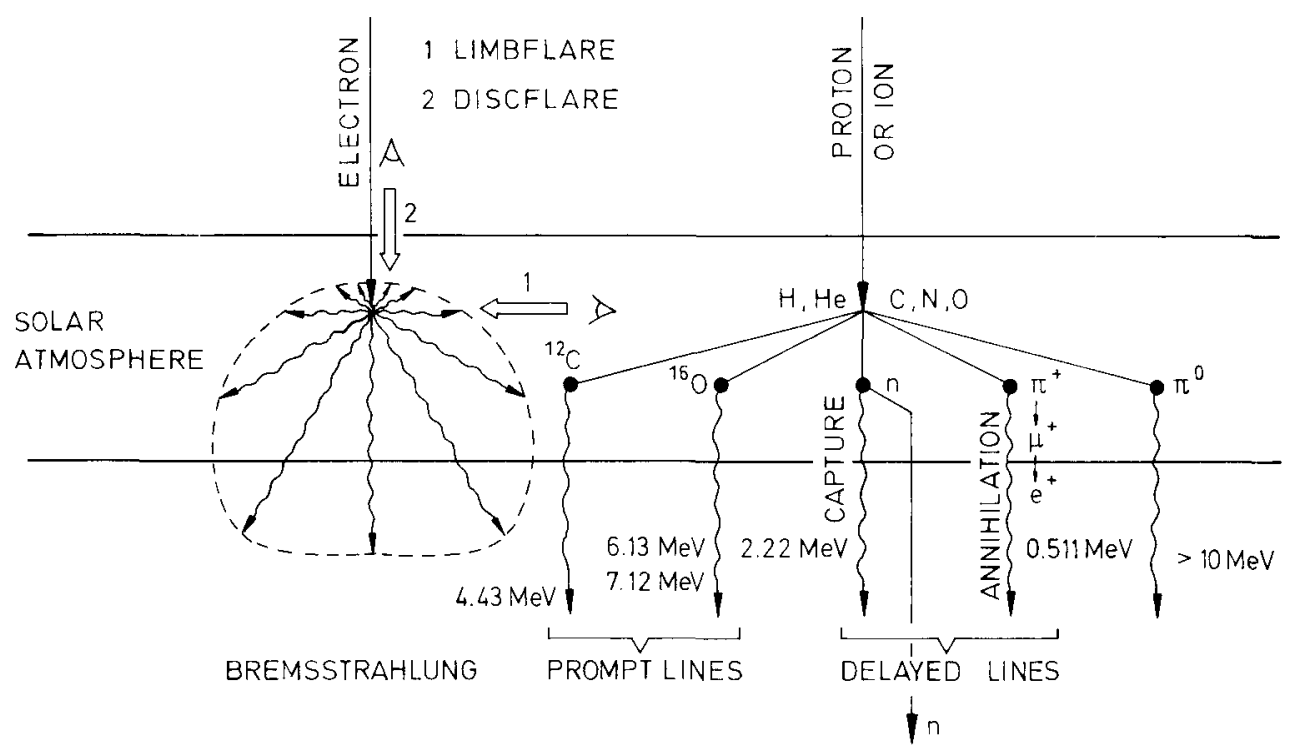

Fig. 1. Schematic drawing of the gamma-ray and neutron production mechanisms. The dashed oval illustrates the anisotropic emission of photons resulting from electron bremsstrahlung. Energy of incident electron $\sim 150 \mathrm{keV}$; photon energy $\sim 50 \mathrm{keV}$ (Elwert and Haug, 1971). The direction given to the arrows symbolizing the gamma rays and neutrons produced by ion interactions does not imply a directivity of the radiation.

\subsection{Electron BREMSSTRAHLUNG}

From the interaction of electrons with matter a continuous spectrum results extending from the energy of the most energetic electrons to almost zero. Using the cross section given by Koch and Motz (1959) for electron-proton bremsstrahlung and the cross section for electron-electron bremsstrahlung given by Haug (1975), Bai (1977) has calculated the photon spectrum resulting from accelerated electrons interacting with a fully-ionized hydrogen plasma. Assuming an electron power-law spectrum, the photon spectrum is also a power law between 10 and $80 \mathrm{keV}$, hardening at higher energies. Above $\sim 400 \mathrm{keV}$ the spectrum is again a power law.

\subsection{ION INTERACTIONS}

Neutral radiation from ion interactions can be divided into three classes, whose relative importance depends upon the energetic particle energy. 


\subsubsection{Nuclear De-excitation Lines}

As opposed to the continuum spectrum of electron bremsstrahlung, narrow gamma-ray lines result from the interaction of accelerated protons and $\alpha$-particles with $\mathrm{He}$ and heavier nuclei in the solar atmosphere. The cross sections for the various nuclear de-excitation lines have been discussed by Ramaty, Kozlovsky, and Lingenfelter (1979). The strongest lines result from the de-excitation of ${ }^{12} \mathrm{C}$ at $4.438 \mathrm{MeV}$ and ${ }^{16} \mathrm{O}$ at $6.129 \mathrm{MeV}$. The proton energies most relevant for the production of these lines are $10-30 \mathrm{MeV}$. Because the lifetimes of the excited states are $10^{-12} \mathrm{~s}$ or shorter, the lines are emitted without a measurable delay, and are thus called prompt lines.

\subsubsection{Neutron Production}

Free neutrons are produced when accelerated ions interact with the ambient atmosphere. The most prolific neutron producing reaction is that of protons on ${ }^{4} \mathrm{He}$ with a threshold of about $30 \mathrm{MeV}$ (Ramaty, Kozlovsky, and Lingenfelter, 1975). These neutrons can have different fates:

- They escape from the Sun and those reaching the Earth before decaying, can be detected with suitable detectors (Chupp et al., 1982; Debrunner et al., 1983; Kocharov, 1983; Chupp et al., 1987). Neutrons leaving the Sun which do not survive can be identified through their energetic decay protons which are trapped by the interplanetary magnetic field (Evenson, Meyer, and Pyle, 1983; Evenson, Kroeger, and Meyer, 1985).

- Neutrons which remain at the Sun can be captured by nuclei before decaying. The reaction ${ }^{3} \mathrm{He}(n, p){ }^{3} \mathrm{H}$ proceeds without the emission of radiation, whereas the reaction ${ }^{1} \mathrm{H}(n, \gamma){ }^{2} \mathrm{H}$ produces the $2.223 \mathrm{MeV}$ line. Because this line is produced in the photosphere it is strongly attenuated in limb flares (Wang and Ramaty, 1974; Hua and Lingenfelter, 1987a). For flares off the limb it is by far the strongest one. As opposed to the de-excitation lines, the $2.223 \mathrm{MeV}$ line is emitted with a delay originating from the finite capture time of neutrons on protons. This is evident in Figure 2 which shows the dynamic spectrum of the flare of 24 April, 1984 in the energy range from 0.3 to $9 \mathrm{MeV}$. The decay of the line can be followed for about $20 \mathrm{~min}$. Because the neutron capture time depends on the proton and ${ }^{3} \mathrm{He}$ densities, the study of the time history of the $2.223 \mathrm{MeV}$ line can give information on the abundance of ${ }^{3} \mathrm{He}$ and the depth in the atmosphere where these reactions take place. Analysis of the decay of the line shows that the capture occurs mainly in the photosphere at hydrogen densities of $\sim 1.3 \times 10^{17} \mathrm{~cm}^{-3}$ (Kanbach et al., 1981; Prince et al., 1983). The ${ }^{3} \mathrm{He} / \mathrm{H}$ ratio derived by Hua and Lingenfelter (1987b) from the $2.223 \mathrm{MeV}$ time history of the flare of 3 June, 1982 is $(2.3 \pm 1.2) \times 10^{-5}$. This value is close to that obtained by Yang et al. (1982) who assume that turbulent mixing of the solar interior does not significantly alter the ${ }^{3} \mathrm{He}$ abundance from that of primordial nucleosynthesis.

\subsubsection{Gamma-Rays from Pion Decay}

Pion production, which requires proton energies of $>100 \mathrm{MeV}$ leads to photons with energies $>10 \mathrm{MeV}$, either through the prompt decay of neutral pions or from the decay 


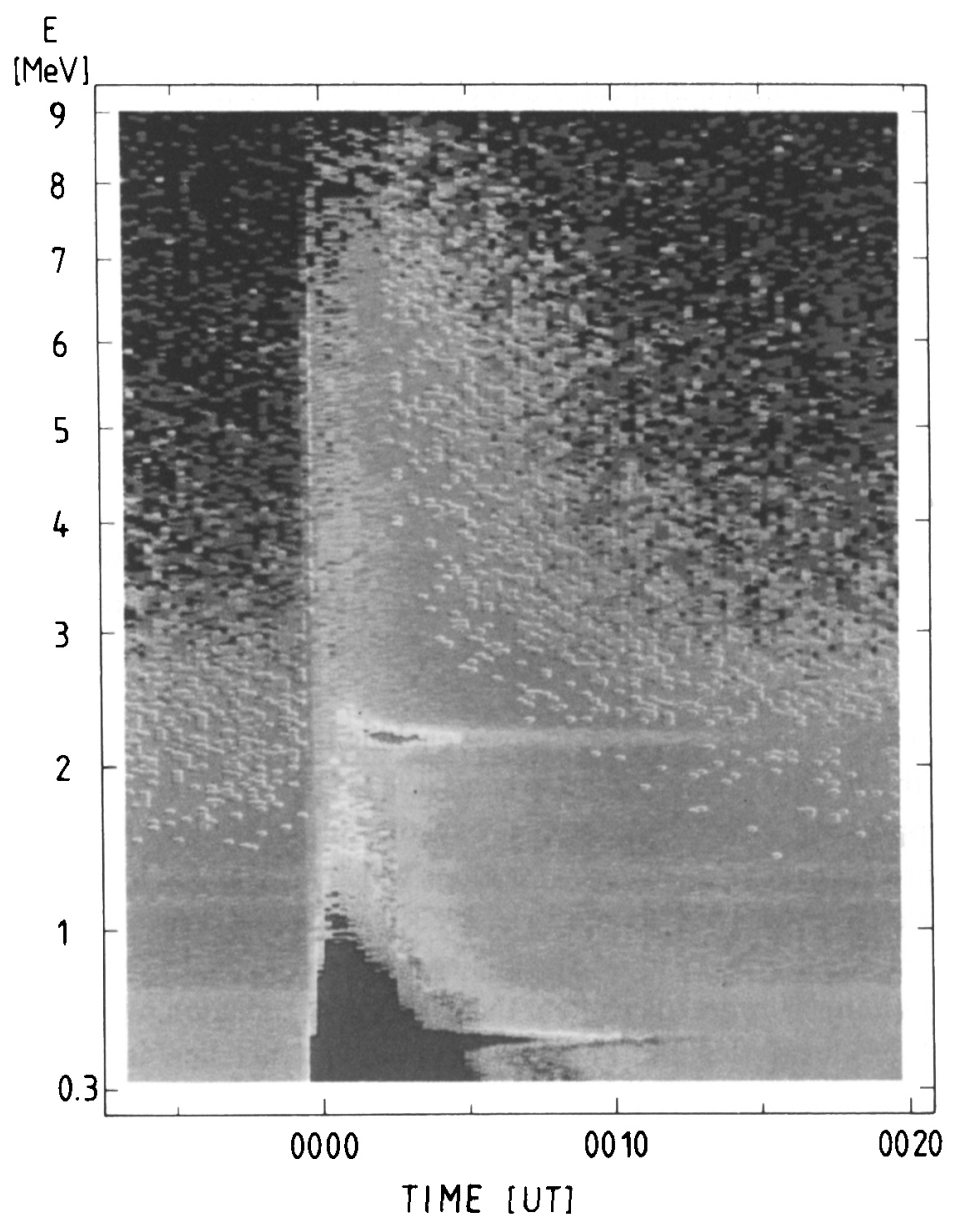

Fig. 2. Temporal evolution of the spectrum of the 24 April, 1984 flare in the energy range $0.3-9 \mathrm{MeV}$ recorded by the gamma-ray spectrometer on SMM. Note the 'afterglow' in the delayed lines at 0.511 and $2.223 \mathrm{MeV}$. The flare is shown without background subtraction.

of charged pions to positrons and electrons. The positrons and electrons slow down mostly due to Coulomb collisions, bremsstrahlung, and synchrotron losses. After thermalization the positrons annihilate with ambient electrons either directly to produce two $0.511 \mathrm{MeV}$ gamma rays per positron or via positronium, where the triplet state $(75 \%)$ decays into 3 photons with energies less than $0.511 \mathrm{MeV}$ (see, e.g., Murphy, Dermer, and Ramaty, 1987). Pion decay positrons have energies of a few tens of $\mathrm{MeV}$, whereas positrons originating from the decay of radioactive nuclei have energies of $\sim 1 \mathrm{MeV}$ (Kozlovsky, Lingenfelter and Ramaty, 1987). Due to the high density of the interaction region the slowing down time of both populations of positrons is short compared to the radioactive decay times, so that the delay seen in Figure 2 reflects the half lives of the parent radioactive nuclei (Murphy and Ramaty, 1984). 


\section{Gamma-Ray Line - Bremsstrahlung Correlation}

It is evident from the foregoing that the solar flare gamma-ray spectrum will appear as a superposition of electron bremsstrahlung, line radiation, and pion decay emission. As pointed out by Ibragimov and Kocharov (1977) and by Ramaty, Kozlovsky, and Suri (1977), above about $1 \mathrm{MeV}$ line radiation will dominate the spectrum. This important fact facilitates the separation of the ionic from the electronic component. To illustrate this, the time-integrated count spectrum of the flare of 7 December, 1982 observed with the Solar Maximum Mission (SMM) Gamma-Ray Spectrometer (GRS) is shown in Figure 3 in the energy range from 0.28 to $9 \mathrm{MeV}$. Between $0.28 \mathrm{MeV}$ (the threshold of the detector) and $1 \mathrm{MeV}$, where strong nuclear lines are absent, the emission is a continuum resembling a power law in spectral shape. Above about $1 \mathrm{MeV}$ the contribution of line radiation, modified by the response of the detector, is apparent. The absense of intense lines above about $7.5 \mathrm{MeV}$ (Crannell, Crannell, and Ramaty, 1979) shows up as a cutoff in the count spectrum. Above $10 \mathrm{MeV}$ (not shown in the figure) a contribution of pion decay gamma-rays and of electron bremsstrahlung has to be considered. To separate the ionic from the electronic component the best fit power-law obtained between 0.28 and $1 \mathrm{MeV}$ is extrapolated to higher energies (dashed line). The

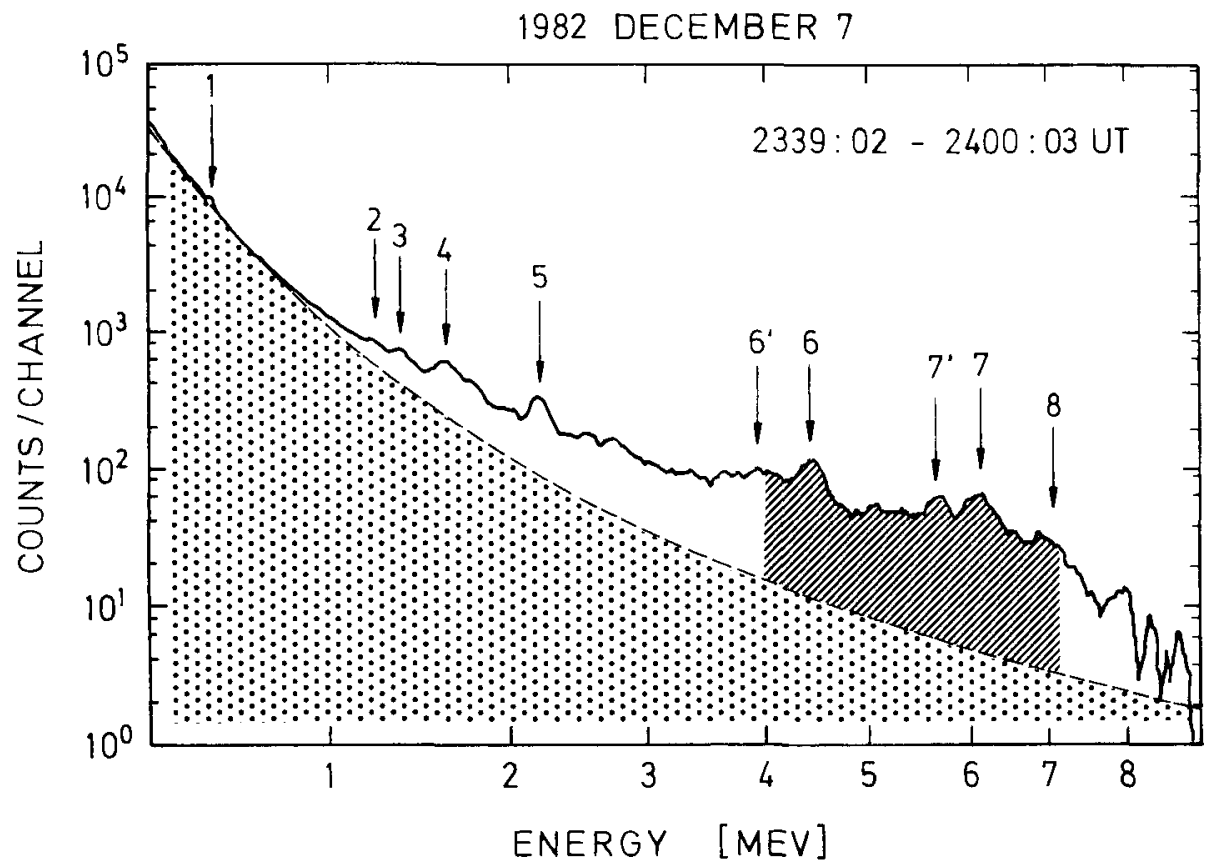

Fig. 3. Time integrated and background subtracted count spectrum of the 7 December, 1982 flare in the energy range $0.28-9 \mathrm{MeV}$. Significant lines and their origin are: (1) $0.511 \mathrm{MeV}$ (annihilation line); (2) $1.24 \mathrm{MeV}\left({ }^{56} \mathrm{Fe}\right)$; (3) $1.38 \mathrm{MeV}\left({ }^{24} \mathrm{Mg}\right)$; (4) $1.63 \mathrm{MeV}\left({ }^{20} \mathrm{Ne}\right)$; (5) $2.223 \mathrm{MeV}$ (neutron capture line, attenuated due to limb proximity of the flare); (6) $4.43 \mathrm{MeV}\left({ }^{12} \mathrm{C}\right) ;(7) 6.13 \mathrm{MeV}\left({ }^{16} \mathrm{O}\right)$, and $(8) 7.12\left({ }^{16} \mathrm{O}\right)$. $\left(6^{\prime}\right)$ and $\left(7^{\prime}\right)$ are the instrumentally produced escape peaks of the lines at 4.43 and $6.13 \mathrm{MeV}$, respectively. The dashed and dotted areas are explained in the text. 
excess above this extrapolation, which is prominent especially between 4 and $7 \mathrm{MeV}$ (dashed area), is ascribed to nuclear interactions.

Since the launch of the SMM satellite in February 1980 until 1987, the GRS has recorded 150 flares with an excess of the time-integrated flux (fluence) at the threshold of the detector $(0.28 \mathrm{MeV}) ; 90$ out of these 150 fiares allow spectral analysis. Of these events $50 \%$ show an excess between 4 and $7 \mathrm{MeV}$. In Figure 4 this excess fluence (dashed area of Figure 3) is plotted versus the fluence above $280 \mathrm{keV}$ (dotted area of Figure 3) attributed to electron bremsstrahlung. For flares without nuclear line excess, the upper limits are indicated. No distinction is made between short impulsive $(<1 \mathrm{~min})$ and long duration events ( $\sim 30 \mathrm{~min})$. The striking feature of Figure 4 is the close correlation between the nuclear line excess, and the $>280 \mathrm{keV}$ continuum fluence, from which the following conclusions are made.

- The smaller number of flares showing observable evidence for nuclear gammaray lines is due only to a lower instrument sensitivity for nuclear emissions $\left(\sim 1\right.$ phot $\left.\mathrm{cm}^{-2}\right)$.

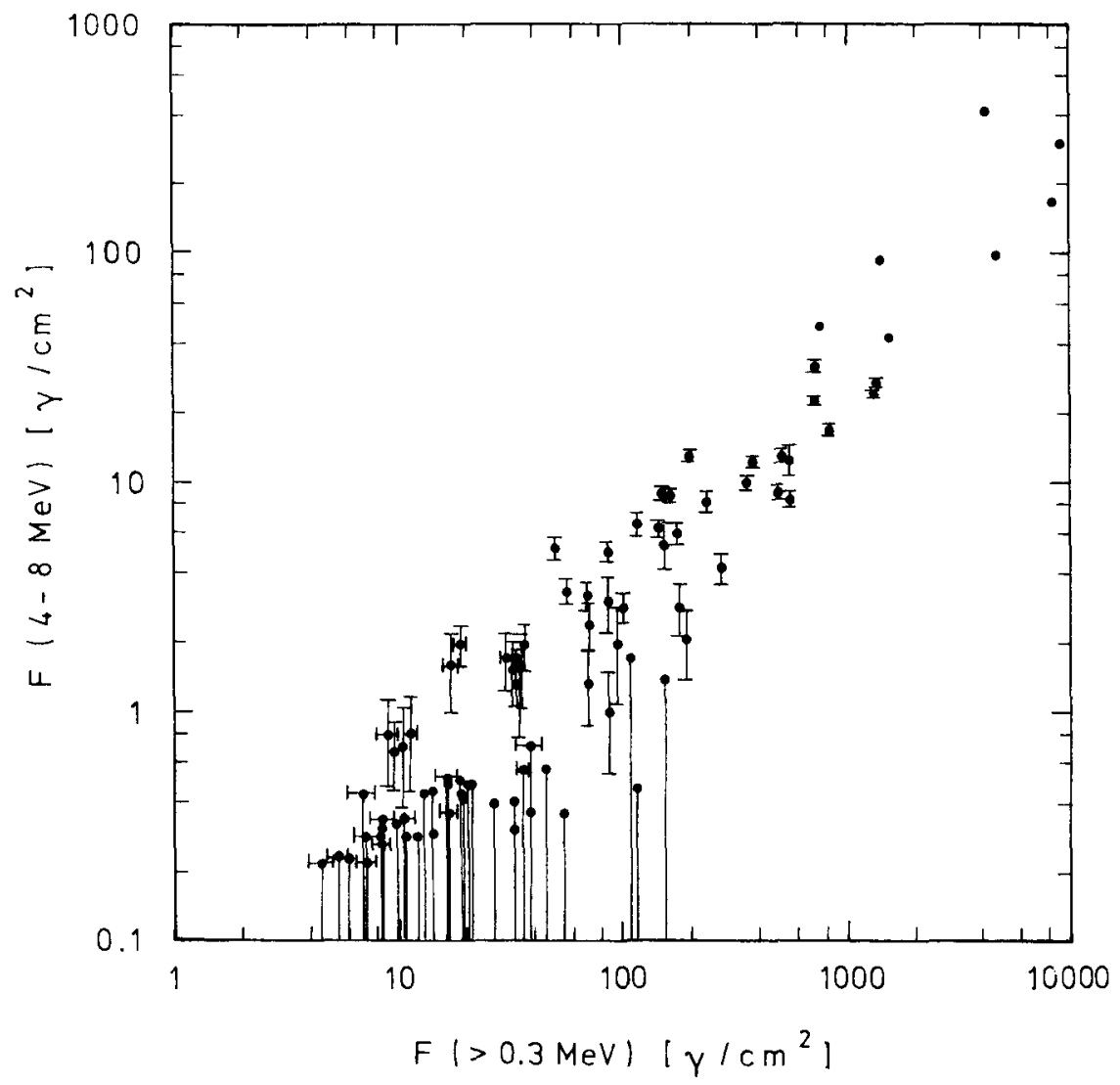

Fig. 4. 4-8 MeV line fluence versus $>0.3 \mathrm{MeV}$ continuum fluence for 90 flares observed by the GRS on SMM (Forrest, private communication). The energy interval $4-8 \mathrm{MeV}$ instead of $4-7 \mathrm{MeV}$ is used to include the ${ }^{16} \mathrm{O}$ line at $7.12 \mathrm{MeV}$. 
- If there is a threshold for the flare size, below which only electrons and no ions can be accelerated, it is below the instrument's sensitivity for nuclear emissions.

- There are no pronounced proton or electron events (missing points in the upper left and lower right of the figure), and

- short impulsive and long-duration events do not appear as separate classes in the diagram. This requires an acceleration mechanism producing high-energy electrons and ions in close proportion independent of the flare duration (Forrest, 1983).

\section{Solar Flare Gamma-Rays and Solar Energetic Particles (SEP)}

When gamma-ray measurements of solar flares became available, it was of interest to examine the relationship between the charged particles producing the gamma-ray emissions at the Sun and those observed in interplanetary space after these flares. In order to minimize the influence of coronal and interplanetary transport effects, it has been proposed to consider only those events which were magnetically well connnected to the observer. Under these conditions the peak flux is a reliable indicator of the particle fluence injected into space (van Hollebeke, Ma Sung, and McDonald, 1975; van Hollebeke, 1979). As the nuclear line fluence is a measure of the number of protons precipitated into the solar atmosphere (Murphy and Ramaty, 1984), a correlation with the peak flux of $10 \mathrm{MeV}$ protons, would be expected.

In Figure 5 the $10 \mathrm{MeV}$ peak proton flux measured with detectors on IMP-8, ISEE-3 and Helios is plotted for 45 well-connected events versus the gamma-ray line fluence obtained directly from the GRS on SMM or inferred from the hard X-ray experiment on ISEE-3 (Cliver et al., 1987). It is evident from the missing points in the lower right hand corner of the figure that large gamma-ray line events also produce large particle fluxes. The converse, however, is not true. There are a number of large particle events for which no nuclear line fluence is measured.

A correlative study of particle events observed with the Helios 1 satellite and the GRS gives a similar result (Kallenrode et al., 1987). This lack of correlation was also recognized in previous investigations (Chambon et al., 1981; von Rosenvinge, Ramaty, and Reames, 1981; Pesses et al., 1981; Yoshimori and Watanabe, 1985). It was pointed out by Cane, McGuire, and von Rosenvinge (1986) and Bai (1986) that the duration of a flare measured at low X-ray energy is an ordering parameter in the sense that longduration events are more prolific in producing interplanetary protons than short ones. The high correlation of big SEP-events with type II radio emission is explained by a shock accelerating the protons high in the corona, where they have ample access to open field lines, whereas in short-duration events the shock acts in low-lying, mostly closed magnetic flux tubes (Pallavicini, Serio, and Vaiana, 1977; Cliver et al., 1987). Coronal shocks, therefore, seem to accelerate predominantly protons (Kallenrode et al., 1987). This conclusion is also substantiated by the facts, that

- for short impulsive events the number of protons interacting in the solar atmosphere is much higher than the number of protons escaping into interplanetary space, whereas 


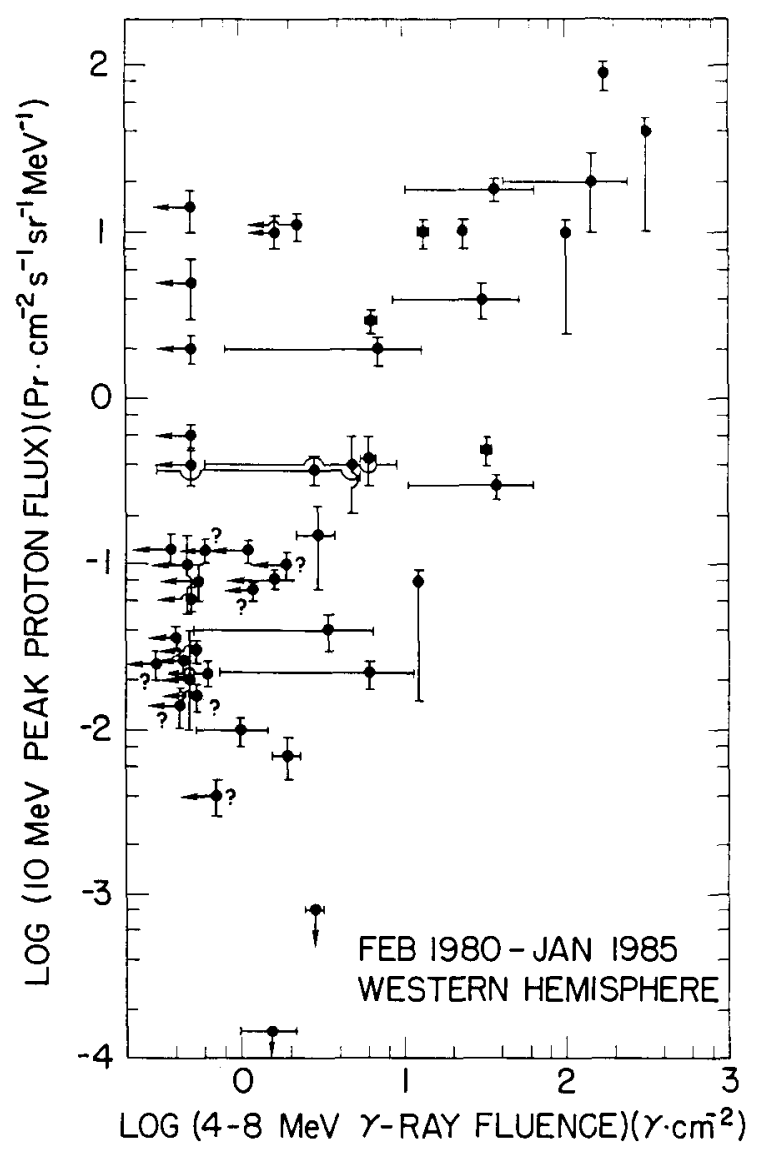

Fig. 5. Peak $\sim 10 \mathrm{MeV}$ proton flux versus 4-8 MeV gamma-ray line fluence for 45 magnetically wellconnected flares (from Cliver et al., 1987).

for long-duration events more particles escape than are precipitated (Ramaty and Murphy, 1987; Hua and Lingenfelter, 1987a), and

- the electron to proton ratio of the escaping particles tends to be higher for flares emitting gamma-rays than for flares which only produce interplanetary particles (Evenson et al., 1984; Cane, McGuire, and von Rosenvinge, 1986).

\section{Proton Energy Spectra}

As already mentioned in Section 2 the relative importance of the emissions caused by nuclear interactions, namely the prompt lines of ${ }^{12} \mathrm{C}$ and ${ }^{16} \mathrm{O}$, the $2.223 \mathrm{MeV}$ capture line and the pion decay radiation, depends upon the energy of the accelerated nuclei. It is, therefore, possible to deduce spectral parameters of the primary particles. Murphy and Ramaty (1984) have calculated the yields of the ${ }^{12} \mathrm{C}$ and ${ }^{16} \mathrm{O}$ de-excitation lines, neutrons, pions, and positrons as a function of the primary proton spectrum under the 


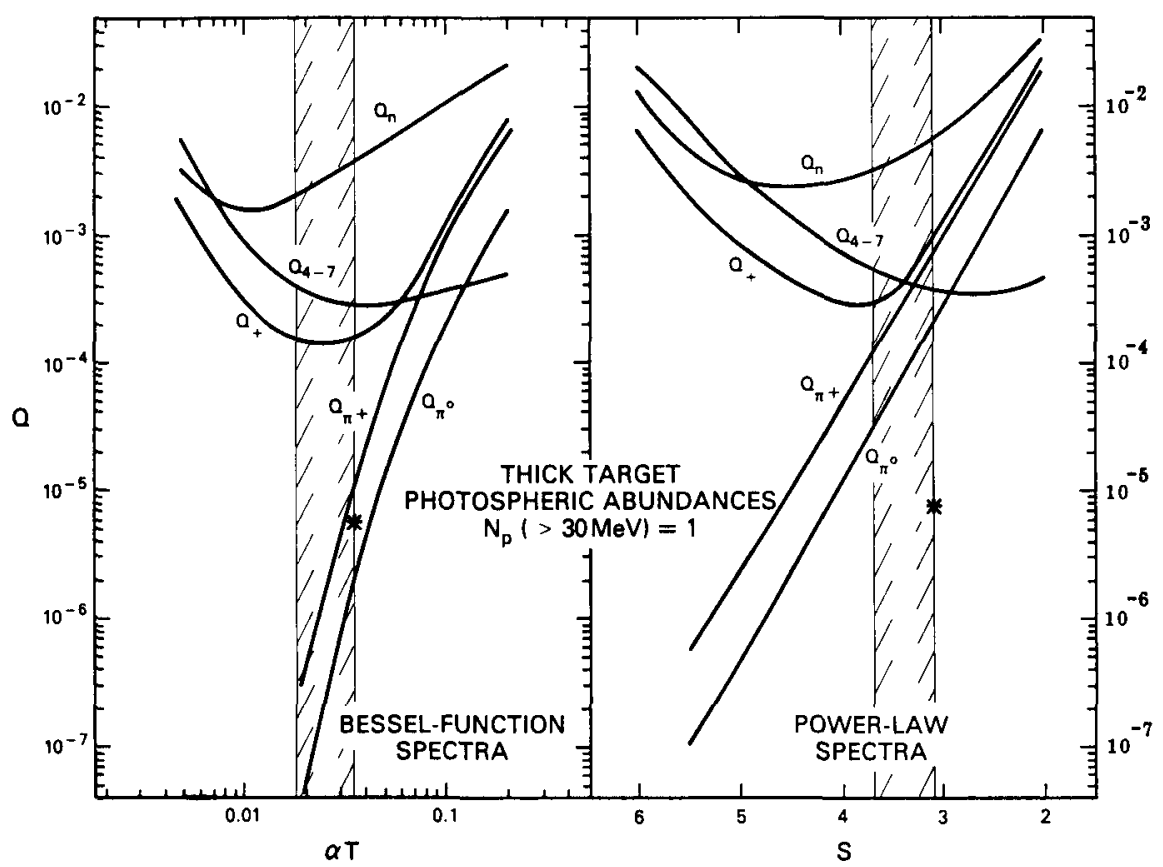

Fig. 6. Neutron, 4-7 MeV nuclear gamma-ray, positron, $\pi^{+}$and $\pi^{0}$ production versus the spectral parameter of a Bessel function spectrum and power-law spectrum for the primary protons (from Murphy and Ramaty, 1984). Indicated by the dashed area is the range of the spectral parameters deduced from observations. Asterisk: $\pi^{0}$-decay fluence of the 3 June, 1982 flare.

assumption that the interacting energetic protons have an isotropic distribution and that they loose all their energy in the ambient medium (thick target case). The results are shown in Figure 6, a reproduction of their Figure 1. The yields are normalized to one proton with an energy $>30 \mathrm{MeV}$. The calculations were performed for a hard and a soft spectrum. On the right-hand side the protons have a power-law spectrum characterized by the exponent $s$. On the left-hand side the proton spectrum has the form of a modified Bessel function of second-order where a high $\alpha T$ indicates a hard spectrum (Ramaty, 1979; Forman, Ramaty, and Zweibel, 1986). The slope of this function increases with increasing energy.

From Figure 6 we see, that the yield of the ${ }^{12} \mathrm{C}$ and ${ }^{16} \mathrm{O}$ lines (here the yield is calculated for the energy band 4-7 MeV, which includes both lines) does not depend very much on the spectral parameters if we exclude very soft proton spectra, whereas the neutron and especially the pion yield increases as the spectrum hardens. To get the $2.223 \mathrm{MeV}$ line fluence, the neutron yield has to be multiplied by the neutron-to$2.223 \mathrm{MeV}$ photon conversion factor. This factor depends upon the spectrum and the angular distribution of the protons and the position of the flare at the Sun (Murphy and Ramaty, 1984; Hua and Lingenfelter, 1987a). The ratio of the $2.223 \mathrm{MeV}$ and 4-7 MeV fluence is a measure of the steepness of the proton spectrum predominantly in the energy range $10-100 \mathrm{MeV}$. It is important to note that because of the different time characteris- 
tics of the prompt lines and the delayed $2.223 \mathrm{MeV}$ line the fluences (time integrated fluxes) rather than the fluxes must be compared. Up to now the fluence ratio for 12 gamma-ray flares has been published (see Hua and Lingenfelter, 1987a). Assuming isotropic distribution of the protons, one obtains $0.018<\alpha T<0.034$ and $3.1<s<3.7$ for the spectral parameters of a modified Bessel function and a power law, respectively. This range of values is indicated in Figure 6 by dashed lines. There is at present only one event, the flare of $3 \mathrm{June}, 1982$, for which also the fluence due to the decay of neutral pions is available. This neutral pion fluence, normalized to the $4-7 \mathrm{MeV}$ fluence, is inserted into Figure 6 by an asterisk, showing that a Bessel function spectral shape of the protons is favoured. This, however, applies only to the first phase of the event (about the spectral hardening during the second phase, see Section 6). There is in fact no flare, for which an unbroken proton power law for the spectrum is suggested by the gammaray measurements. Even the ground level event of 7 December, 1982 is no exception (Rieger et al., 1987).

If we try to deduce proton-energy spectra from the particle observations in space, we have to bear in mind that particles originating from different phases of a flare, implying different acceleration mechanisms, can be mixed together in the signal (Neustock, Wibberenz, and Iwers, 1985). In this respect photon measurements are superior to particle measurements, because different phases can generally be distinguished by their temporal characteristics (see Figure 8). For charged particles the influence of coronal propagation and interplanetary transport must also be considered. As already mentioned, these effects can be minimized by using particle measurements of magnetically well-connected flares. McGuire and von Rosenvinge (1984) have reviewed the measurements of such well-connected events and found that the spectra can be best approximated with a Bessel function. For protons the $\alpha T$-values are within the same range as those obtained from the gamma-ray data. These observations suggest, that for most flares a common mechanism could accelerate both particle populations. It must, however, be pointed out that there are only 4 flares for which spectral information both from gamma-ray (GRS) and from particle measurements exists. These are the events of 7 June, 1980 (McGuire and von Rosenvinge, 1984); 21 June, 1980; 3 June, 1982 (McDonald and van Holleke, 1985); and 7 December, 1982 (Rieger et al., 1987). A Bessel function can be fit to the particle data of the flares of 7 June, 1980 and 21 June, 1980 , in accord with the results of the gamma-ray measurements. The particle spectra of the flares of 3 June, 1982 and 7 December, 1982, however, are too hard to be fit with a Bessel function. They could, therefore, result from a different acceleration mechanism operating during a second phase (see Ramaty and Murphy, 1987).

Shortly after the reports of ground-level cosmic-ray enhancements (Ground Level Events) following solar flares, Biermann, Haxel, and Schlüter (1951) conjectured that the $\mathrm{GeV}$ protons at the Sun could produce neutrons observable at Earth. Neutrons were detected by the GRS and ground based neutron monitors after the flares of 21 June, 1980 and 3 June, 1982. Because these detectors do not measure the neutron energy directly, it must be derived from the time of flight between the Sun and Earth. If the duration of a flare, determined by the emission time history in the energy band 4-7 MeV 
and/or at $>25 \mathrm{MeV}$ is short compared to the flight time between Sun and Earth of the fastest neutrons, the neutron production can be approximated by a delta function in time, coincident with the peak of the photon emission. Applying this criterion to the neutron flare observations of 21 June, 1980 and 3 June, 1982, Murphy and Ramaty (1984) and Hua and Lingenfelter (1987c) show that a good fit of the calculated neutron flux time history with the data is achieved when a Bessel function spectral type instead of a power law is assumed for the primary protons. The spectral parameters $\alpha T$ are in good agreement with the values obtained from the ratio of the $2.223 \mathrm{MeV}$ line - and 4-7 MeV fluence during the impulsive phase. They point out that the bulk of the neutrons is a product of the impulsive phase. On the other hand Chupp et al. (1987), by analyzing the GRS and neutron monitor data, find that the majority of the neutrons is produced after the impulsive phase. This discrepancy is further discussed by Ramaty and Murphy (1987).

\section{Temporal Characteristics of Flares}

In addition to the energy spectra of the primary particles, deduced from gamma-ray line, neutron, and charged particle measurements, the time history of a flare in different energy bands is an important observational ingredient setting constraints on particle acceleration mechanisms.

Before gamma-ray measurements were available, Wild, Smerd, and Weiss (1963) used radio data in their search for the origin of the flare-energy release. From the observation that impulsive meter-wave type III bursts were followed after several minutes by type II meter-wave emissions, indicative of a shock wave moving through the solar corona, they developed a concept of two acceleration phases. According to this idea, electrons should be accelerated impulsively during phase one to energies of $\sim 100 \mathrm{keV}$. The second more gradual phase, which needs phase one as a trigger, then accelerates electrons to relativistic energies and creates high-energy ions producing ground level events. This concept of two phases or two steps (Bai and Ramaty, 1979) of particle acceleration during solar flares was basically accepted until the early 1980's, when flare measurements with high time resolution in the $\mathrm{X}$ - and gamma-ray regime became available through the SMM- and Hinotori-detectors. Among the many flares with gamma radiation, events were observed with impulsive emission occurring simultaneously in X- and gamma-rays within the 1-2 s time resolution of the instruments (Rieger, 1982; Forrest and Chupp, 1983; Rieger et al., 1983; Yoshimori et al., 1983; Kane et al., 1986). To stress this important observational point, the time history of the flare of 7 May, 1983 is shown in Figure 7. This intense event consists mainly of one impulsive burst of about 1 min duration visible in three decades of energy. The emission seen in panel 1 (time resolution $1 \mathrm{~s}$ ) is bremsstrahlung of subrelativistic electrons. The emission in panel 2 (time resolution $2 \mathrm{~s}$ ) which appears more bursty than that at lower energies, originates mainly from the nuclear de-excitation lines of ${ }^{12} \mathrm{C}$ and ${ }^{16} \mathrm{O}$ at 4.4 and $6.1 \mathrm{MeV}$, respectively (see Section 2). Above $10 \mathrm{MeV}$ primary electron bremsstrahlung is again the dominant radiation mechanism, unless the primary proton 


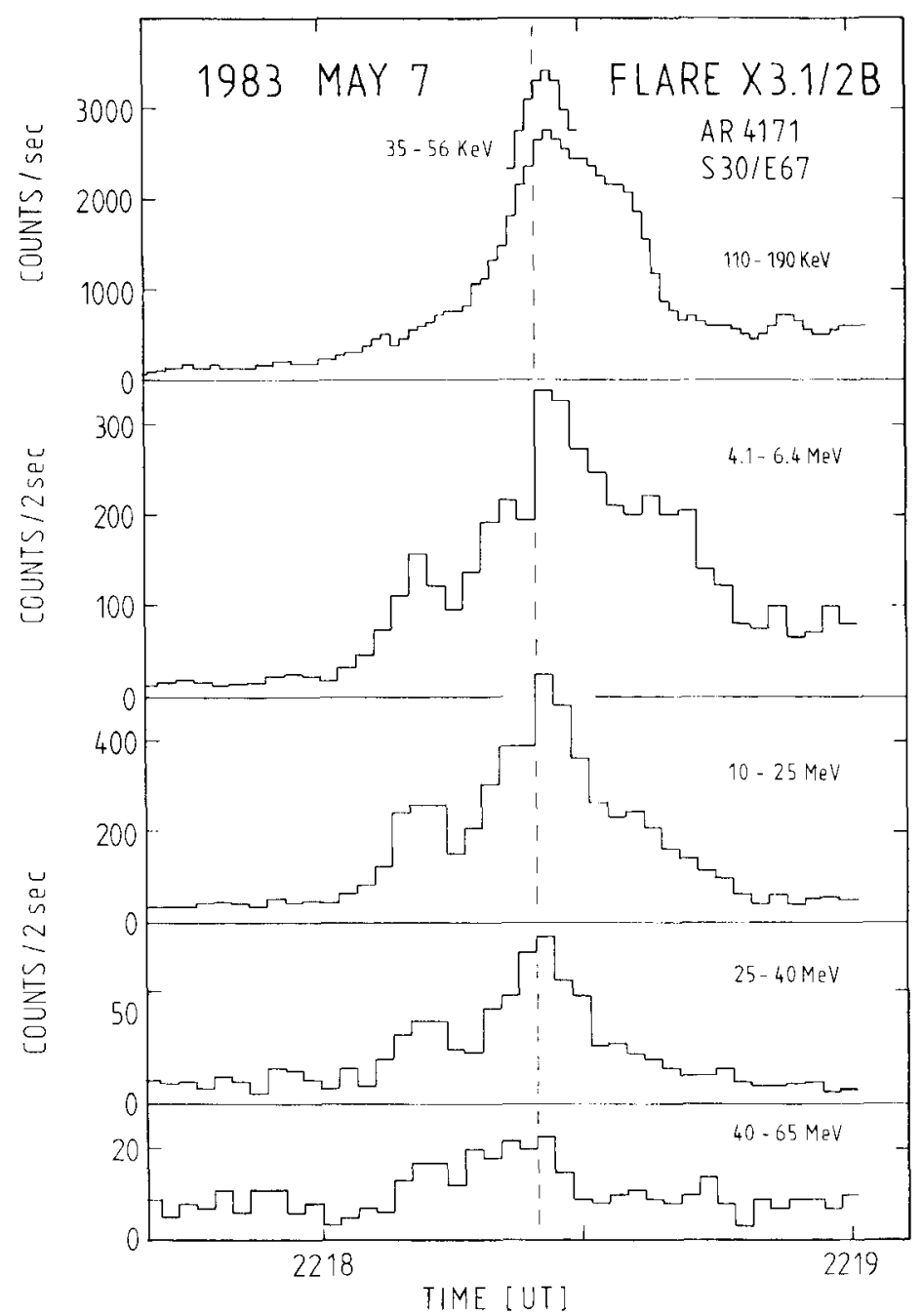

Fig. 7. Time history of the 7 May, 1983 flare in different energy bands (SMM GRS).

spectrum is very hard (Rieger et al., 1983; Ramaty et al., 1983). It is evident from Figure 7 that the peak of the emission at gamma-ray energies occurs simultaneously within the limit of the time resolution with respect to the peak of the medium energy $\mathrm{X}$-rays, usually taken as the reference time of the impulsive phase (Bai and Ramaty, 1979). Strangely enough, there is a precursor preceding the main peak, which is prominent only in gamma-rays, but not in X-rays. We will, however, not refer to this curiosity. These observations show, that ions and electrons can be accelerated impulsively ( $\sim 1 \mathrm{~s}$ ) to energies of $>10 \mathrm{MeV}$ and $\sim 100 \mathrm{MeV}$, respectively. If we take into account that the emission versus time is a complicated superposition of acceleration-, transport-, and energy-loss processes and that the last two have a tendency to smooth out quick temporal changes, the acceleration by itself may even be more bursty in time. 
Under rare conditions ions (protons) can be accelerated to greater energies during the impulsive phase. This is evident from the analysis of the flare of 3 June, 1982 (Figure 8). This very intense event had a distinctive 2-phase nature at energies $>10 \mathrm{MeV}$ : an impulsive emission of $\lesssim 1$ min duration was followed by a more gradual one lasting several minutes. The primary peak spectrum shows a flattening above $40 \mathrm{MeV}$ which has been attributed to neutral pion decay photons (Forrest et al., 1985, 1986). Measurements from the Jungfraujoch neutron monitor show that high energy neutrons $(\sim 1 \mathrm{GeV})$ were produced already during the impulsive phase (Debrunner et al., 1983; Chupp et al., 1987). These observations imply that during the first or impulsive phase protons can be accelerated to $\mathrm{GeV}$-energies.

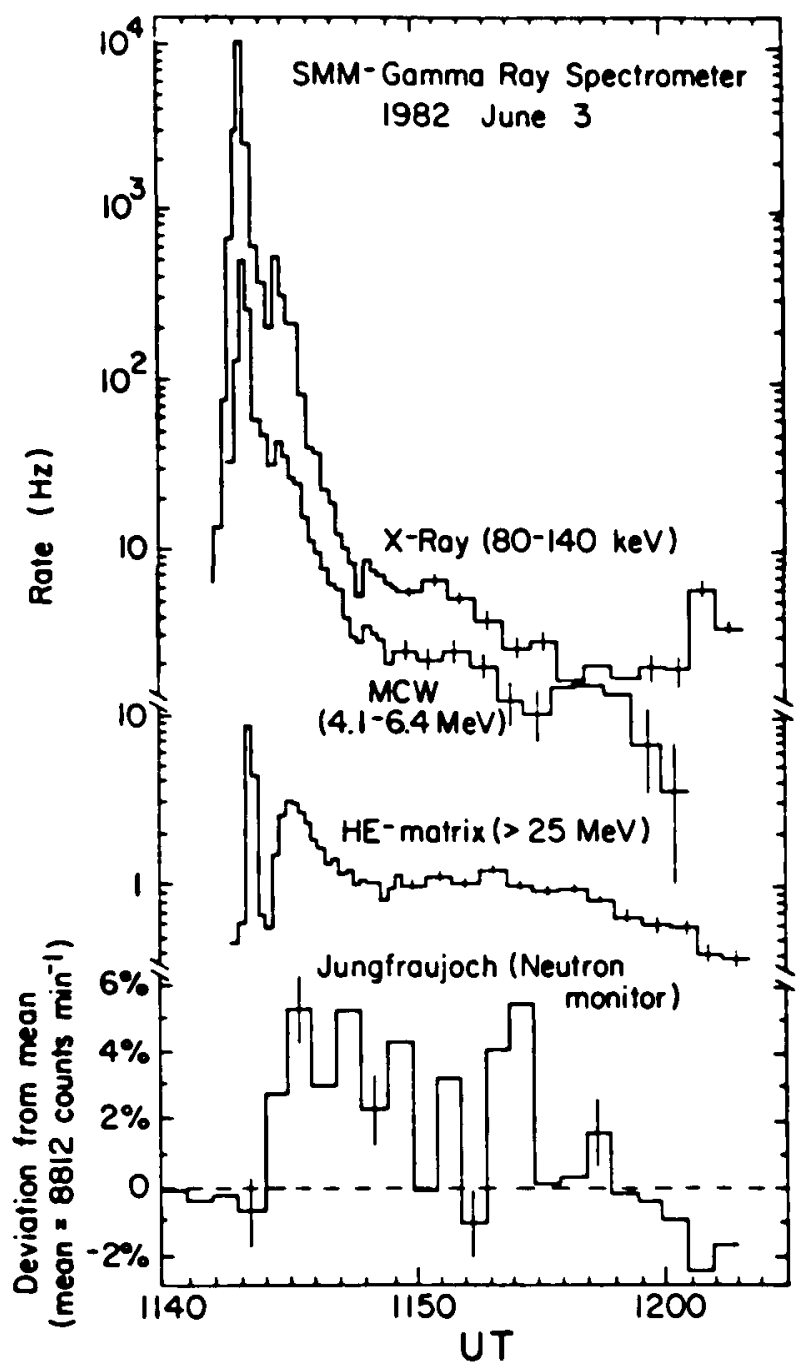

Fig. 8. Time history for several data channels of the SMM GRS and of the Jungfraujoch neutron monitor count rate for the 3 June, 1982 flare (from Chupp et al., 1985). 
The SMM and Hinotori spectrometers and detectors on earlier missions recorded numerous other flares, where a delay between the emissions at lower $(\sim 50 \mathrm{keV})$ and higher energies was observed (see, e.g., Vlahos et al., 1986). While these delays have been interpreted as evidence for a second step (or second stage) acceleration (Bai and Ramaty, 1979; Bai, 1982; Bai et al., 1983a, b; Bai and Dennis, 1985), a trap plus precipitation model (Kane, 1974; Melrose and Brown, 1976; Bai and Ramaty, 1979; Vilmer, Kane, and Trottet, 1982; MacKinnon et al., 1983; Ryan, 1986) and a trap plus turbulent propagation model (Bespalov, Zaitsev, and Stepanov, 1987), a more direct observational proof for a second phase acceleration comes from the gamma-ray measurements of the 3 June, 1982 flare. As already mentioned a gradual peak $(>10 \mathrm{MeV}$ ) appears after the first intense burst, which does not stand out at X-ray energies and in the nuclear energy band. The spectrum of this second peak is harder than that of the first impulsive burst, showing a large contribution of $\pi^{0}$-decay photons (Forrest et al., 1985). This second peak together with particle measurements in space after the flare (McDonald and van Hollebeke, 1985) are interpreted by Murphy, Dermer, and Ramaty (1987) as a second phase acceleration by a shock wave going through the solar corona. Another event with similar temporal characteristics is the flare of 24 April, 1984 , for which a detailed evaluation of the spectrum is not yet available. It is of interest to note that these two flares are by far the most intense events observed by a detector sensitive to energies above $10 \mathrm{MeV}$. Thus, from the gamma-ray measurements the two phase or two-step acceleration process during solar flares is not ruled out. The important new finding, however, is that electrons can be accelerated impulsively $(\sim 1 \mathrm{~s})$ in a single step to $\sim 100 \mathrm{MeV}$ and ions to $\mathrm{GeV}$ energies within seconds. This 'inconvenience' has to be taken into account, when one deals with acceleration theories.

\section{Acceleration Mechanisms}

Any viable acceleration mechanism should be able to account for the time-scales of electron and proton acceleration, their energy spectra, the total number or ratio of accelerated particles and it must be capable of imparting a large fraction of the available flare energy to the energetic particles. The mechanisms, most widely investigated, are stochastic acceleration, shock acceleration and acceleration by DC electric fields (for a review see Forman et al., 1986; Scholer, 1988; Cargill and Vlahos, 1989; and Sturrock, 1989).

\subsection{STOCHASTIC ACCELERATION}

This process is essentially second-order Fermi acceleration, where particles in a turbulent plasma change their energy in a random way with many increases and decreases in energy. Various wave-particle interactions have been considered, for instance resonant pitch angle scattering from Alfvén waves, interaction with magnetosonic waves (Forman et al., 1986) and scattering by whistler turbulence (Miller and Ramaty, 1987). By assuming that the acceleration efficiency $\alpha$ and the average time $T$ during which the particles experience acceleration are energy independent, it was shown, that the particle 
energy spectrum can be represented by a modified second-order Bessel function in the nonrelativistic regime, and by a power law in the ultra relativistic regime (Ramaty, 1979). In both cases the product of the parameters $\alpha$ and $T$ characterizes the shape of the spectra in the sense that a larger value of $\alpha T$ corresponds to a harder spectrum.

For the nonrelativistic case $\left(E \ll m_{0} c^{2}\right)$ and at energies $E \gg 3.26(\alpha T)^{2} \mathrm{MeV}$ nucl. $^{-1}$ the formula

$$
\frac{\mathrm{d} J}{\mathrm{~d} E}=E^{3 / 8} \exp \left(-\left(E /\left(3.26(\alpha T)^{2}\right)\right)^{1 / 4}\right),
$$

where $\mathrm{d} J / \mathrm{d} E$ is the differential particle energy spectrum, is a good approximation to the modified Bessel function (Forman et al., 1986). In the ultrarelativistic case the powerlaw exponent is given by

$$
\gamma=\frac{1}{2}(9+12 / \alpha T)^{1 / 2}-\frac{1}{2} .
$$

Using this model, energy spectra of protons and $\alpha$-particles observed in space were successfully fitted (Ramaty, 1979). As already mentioned in Section 5 the spectral parameter $\alpha T$ deduced from gamma-ray measurements varies in narrow limits between 0.018 and 0.034 . If these values are used to fit the electron spectra above $1 \mathrm{MeV}$ by using Equation (2), we get a slope which is much steeper than observed (e.g., Ramaty, 1979; Evenson, Kroeger, and Meyer, 1985; Dröge et al., 1989).

The acceleration time in stochastic acceleration was investigated by Miller, Ramaty, and Murphy (1987). It is shown that the time to accelerate ions from ambient energies to $5,30,400$, and $6000 \mathrm{MeV}$ nucl. ${ }^{-1}$ is $1,2,6$, and $16 \mathrm{~s}$, respectively. This is not in serious contradiction to what can be deduced from the temporal characteristics of gamma-ray flares. A problem, however, arises with the time-scale implied by the observations for electron acceleration. To accelerate them to tens of $\mathrm{MeV}$ in the same acceleration region as the ions takes about $100 \mathrm{~s}$, which is much too slow (Ramaty and Murphy, 1987). The time-scales are shorter if electron acceleration due to whistler turbulence is taken into account. Miller and Ramaty (1987) showed that in this case electrons can be accelerated to $100 \mathrm{MeV}$ in about $10 \mathrm{~s}$. Similar time-scales are obtained by Dröge and Schlickeiser (1986) who investigated the combined effects of first- and second-order Fermi acceleration. But in view of the simultaneous peaking (1-2 s) of the emission at $\sim 50 \mathrm{keV}$ and $\sim 50 \mathrm{MeV}$ observed in some flares (see Section 6) there still remain doubts if stochastic acceleration is a mechanism suitable for the impulsive phase.

\subsection{Diffusive SHOCK ACCELERATION}

In this case the basic acceleration mechanism is the first order Fermi process with a turbulent medium on both sides of a quasi parallel shock. The particles are scattered by the inhomogeneities so that they experience multiple accelerations at the shock. When losses are due only to convection of the particles away from the shock downstream and the shock size is large with respect to particle diffusion lengths (planar shock), the particle spectrum deduced in this case is a power law in momentum space with an 
exponent determined by the compression ratio $r$ of the shock (Blandford and Ostriker, 1978). The corresponding differential particle intensity, $(\mathrm{d} J / \mathrm{d} E)_{0}$, in units of particles $\mathrm{cm}^{-2} \mathrm{~s}^{-1} \mathrm{sr}^{-1} \mathrm{MeV}^{-1}$ is given by (Ellison and Ramaty, 1985)

$$
(\mathrm{d} J / \mathrm{d} E)_{0} \sim\left(E^{2}+2 E m_{0} c^{2}\right)^{-\gamma},
$$

where $m_{0} c^{2}$ is the rest mass energy and $\gamma=\frac{1}{2}(r+2) /(r-1)$. Several effects can truncate this power-law behaviour at high energies, for instance shock life times comparable to particle acceleration times (Forman, 1981) and shock sizes comparable to particle diffusion length (e.g., Ellison, 1984). If one incorporates these effects, then Equation (3) is replaced by

$$
\mathrm{d} J / \mathrm{d} E \sim(\mathrm{d} J / \mathrm{d} E)_{0} \exp \left(-E / E_{0}\right),
$$

where $E_{0}$ is called the turnover kinetic energy, above which the particle spectrum steepens considerably. Using this two-parameter equation Ellison and Ramaty (1985) were able to fit particle energy spectra of several flares. It is important to note, that for flares where electron and proton measurements are available, a satisfactory fit to the spectra of both types of particles is obtained by using the same values for the parameters $r$ and $E_{0}$. This formula was also applied successfully to ground level events. The differential proton energy spectrum measured after the 3 June, 1982 flare (McDonald and van Hollebeke, 1985) was fitted by Equation (4) with $\gamma=1.7$ and $E_{0}>300 \mathrm{MeV}$. Using these values and the time history of the $0.511 \mathrm{MeV}$ annihilation line (Share et al., 1983) it is possible to model the high-energy emission during the second phase of this event (Ramaty, Murphy, and Dermer, 1987).

Ellison and Ramaty (1985) point out that shock acceleration not only can explain the low $e / p$ ratio observed at high energies, but also the short acceleration times for electrons and protons implied by the gamma-ray measurements. If the background turbulence is strong enough to produce scattering meanfree paths that are less than about 100 times the gyroradius, electrons and protons can be accelerated to $100 \mathrm{MeV}$ in about $1 \mathrm{~s}$. With the appropriate choice of the shock compression ratio $r$ and the turnover kinetic energy $E_{0}$ shock acceleration could thus be a candidate also for the impulsive phase provided the time to generate the shock, or the shocks, is short $(<1 \mathrm{~s})$.

Papadopoulos et al. (1985) and Decker and Vlahos (1986) have investigated the acceleration of charged particles by oblique turbulent shocks through numerical simulations. In their model a sudden energy release heats the plasma in some volume and a shock is formed in a few microseconds. The ions in the tail of the heated plasma serve as injected particles, which are then accelerated most effectively by quasiperpendicular shocks to energies of $50 \mathrm{MeV}$ in less than milliseconds. In this treatment, however, the acceleration of electrons has not been addressed.

Quasi-perpendicular fast magnetosonic shock waves have been invoked by Ohsawa and Sakai (1987) and Sakai and Ohsawa (1987) as an acceleration mechanism for ions and electrons to relativistic energies in a short enough time to explain the gamma-ray observations of the 3 June, 1982 flare. The authors point out that in loops with strong magnetic fields (low-lying loops) magnetosonic waves could lead to impulsive ion and 
electron acceleration (first or impulsive phase). As the shock propagates out to weak magnetic field regions, strong electron acceleration ceases, while ion acceleration continues (second or gradual phase). But if we apply this model to the temporal characteristics of the flare (see Figure 8) one would expect to see a smooth transition above $25 \mathrm{MeV}$ from phase 1 to phase 2 . The difference in the temporal and spectral characteristics of both peaks, however, makes it tempting to assume that two physically different acceleration mechanisms were in operation causing an impulsive and a gradual phase of the flare (see Ramaty and Murphy, 1987). It is of interest to note that Kocharov et al. (1988) interpret the time history by a single acceleration process occurring during the impulsive peak. To explain the second maximum at $>25 \mathrm{MeV}$ a fast rise of plasma turbulence is assumed to occur at the end of the impulsive peak, leading to precipitation due to pitch angle scattering of impulsively created high-energy protons.

\subsection{ELECTRIC FIELDS}

In addition to stochastic processes there is also the possibility to accelerate particles in direct electric fields (see, e.g., Forman, Ramaty, and Zweibel, 1986). Two approaches invoking DC electric fields, which have their routes in the investigation of auroral phenomena, shall be briefly considered here. It has been shown with ion cloud experiments carried out in the auroral zone (Haerendel et al., 1976; Wescott et al., 1976) and by measurements made with the S3-3 satellite (Mozer et al., 1980; Temerin et al., 1982) that under certain conditions electrostatic potential drops along the magnetic field lines can develop, by which auroral particles are accelerated. A necessary condition for this process to operate is the existence of intense field aligned currents exceeding a critical value (e.g., Spicer, 1982).

Assuming, that this magnetic field parallel potential drop is located over a very short parallel distance (supported by laboratory measurements) Carlquist (1986) has shown that the maximum potential drop attained by this 'double layer' is a function of the total current rather than the current density. In the solar atmosphere, where currents of the order $10^{11}-10^{12} \mathrm{~A}$ are known to exist (Anzer and Tandberg-Hanssen, 1970) he obtains $\sim 10^{5} \mathrm{MV}$ for the maximum potential drop of the double layer, which would be enough to account for even the highest energy particles observed. The time to accelerate the particles is a fraction of a second provided the double layer attains the full power in a short time-scale.

In the model of Haerendel (1987) the flare is explained by a strong twisting and subsequent untwisting of a large number $\left(\sim 10^{5}\right)$ of narrow flux tubes, which are thought to be embedded in an active region. The sudden release of the flare energy which is triggered by an unidentified cause (e.g., new flux emergence, reconnection in the corona, etc.) leads to a partial reinvestment of the flux tubes which can leave behind even more highly twisted ones. The corresponding currents create the low temperature flare in the lower chromosphere by classical Joule heating. In a small minority of flux tubes the current reaches values that makes them unstable and cause strong field aligned potential drops. One $\mathrm{GV}$ and more can be attained. In sufficiently strong primary fields $(\sim 2000 \mathrm{G}$ ) the 'reverse current' problem (e.g., Colgate, 1978) does not arise in this 
model, because the currents are assumed to be contained in small scale structures with random orientation. Thus, the net current can be many orders of magnitude below the sum of all the current filaments. It is pointed out, however, that the model rests upon the ability of the flux tubes to be stable against a twisting of many revolutions over the length of the tube.

\section{Evidence for Beaming of the High-Energy Particles?}

Acceleration by DC electric fields leads to particle beams directed along the magnetic field lines, whereas stochastic acceleration processes have a tendency to create isotropic particle distributions. Investigation of anisotropies in the velocity distribution of flare generated particles can, therefore, provide important clues about particle acceleration and about the particle transport in the flaring loops. The directionality of electrons can be studied, because bremsstrahlung photons are emitted anisotropically in the direction of motion of the electrons (see Figure 1), increasing in anisotropy with increasing electron energy (Elwert and Haug, 1971). If the electrons move predominantly downward towards the Sun (pencil beam) or tangential to the solar surface due to mirroring in the converging magnetic fields (fan beam), a flare observed near the limb will appear brighter than a flare observed at the disc. Weak flares are, therefore, detectable only if they occur close to the limb. But all efforts to show this 'limb brightening' by using X-ray flares as a proxy did not lead to a positive result (Kane, 1974; Datlove et al., 1977). This, however, does not rule out the existence of beams, because electrons of the solar atmosphere scatter the photons back (Compton backscattering), thereby smearing out any anisotropy of the primary radiation (Bai and Ramaty, 1978). A limb brightening begins to show up if we mark all the SMM GRS flares with known position at the solar disc. The number of flares close to the limb is higher than is expected for isotropically emitting flares (Vestrand et al., 1987). The finding that the best fit power-law electron continuum between 0.28 and $1 \mathrm{MeV}$ is harder for flares near the limb than for flares closer to the disc center can be interpreted as an additional indication of a beaming of the electrons. Spectral hardening in the energy range 50-300 keV towards the limb was also found in a sample of flares observed by the Venera 13 spacecraft (Bogovalov et al., 1985). Evidence for a directivity is also given by Kane et al. (1988) in their analysis of flares observed stereoscopically by the ISEE-3 and Pioneer-Venus orbiter in the energy range $0.1-1 \mathrm{MeV}$. A third method is used by Bai (1988). Instead of counting the number of limb and disc flares he plots the fluence of the GRS flares after normalization with respect to the total counts measured by the hard X-ray burst spectrometer (HXRBS) on SMM versus the heliocentric angle and finds a systematic increase of the luminosity of the GRS flares towards the limb. The most favourable energy band to investigate the directivity of the electrons is between 8 and $40 \mathrm{MeV}$, where the flare radiation is predominantly electron bremsstrahlung. At these high energies the Compton backscatter is negligible and the bremsstrahlung cross-section is highly anisotropic. Figure 9 shows the $\mathrm{H} \alpha$-position at the Sun of the flares with photon emission above $10 \mathrm{MeV}$. A concentration towards the limb is clearly visible (Rieger et al., 1983; Canfield et al., 


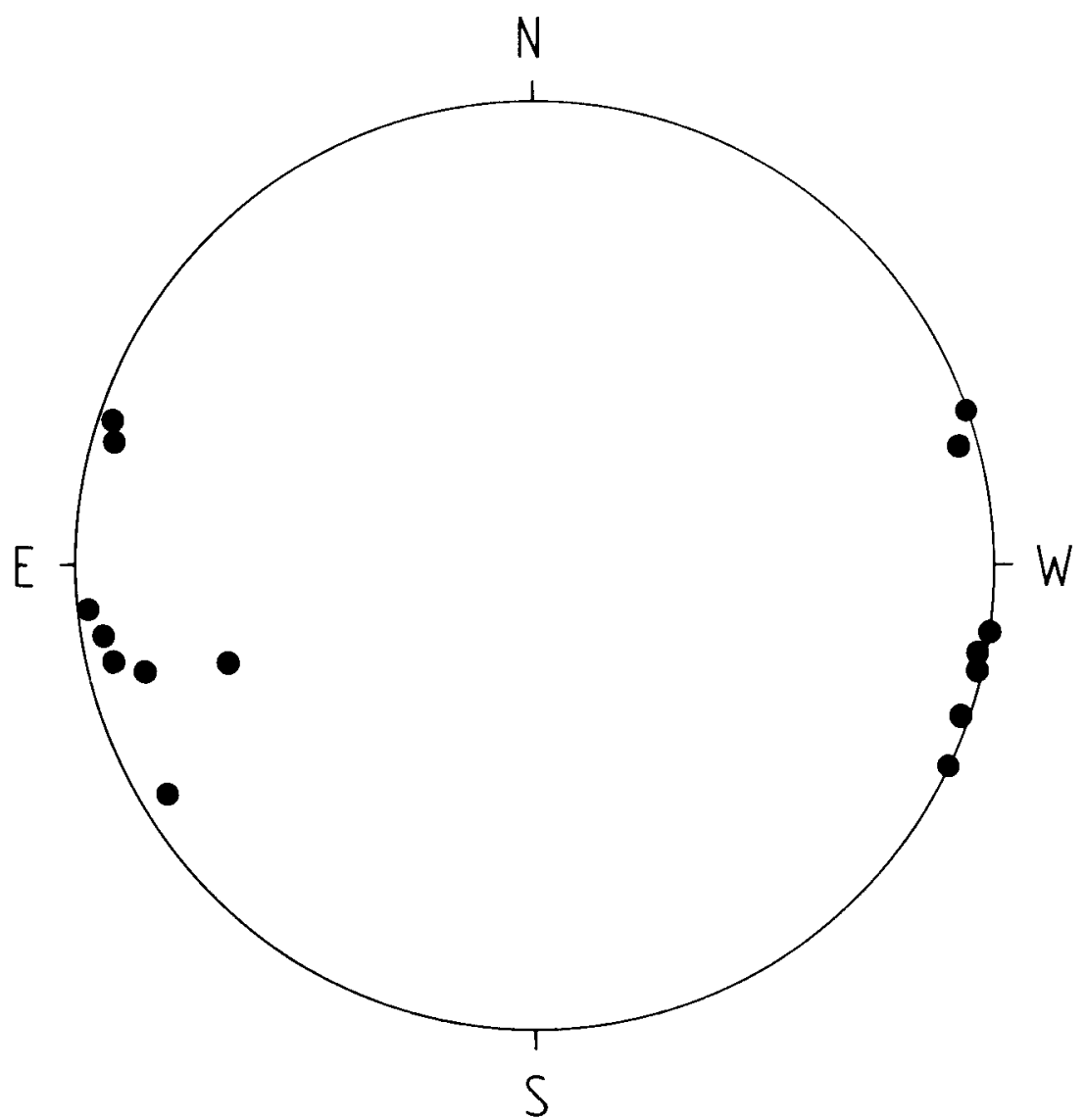

Fig. 9. Position of flares with emission above $10 \mathrm{MeV}$ at the solar disc observed by the GRS on SMM.

1986). Only two out of 15 events are at heliocentric angles $<64^{\circ}$. Although the number of flares with emission $>10 \mathrm{MeV}$ is still small, this observational fact has led to theoretical investigations about the directionality of the primary high-energy electrons.

Naively interpreted one could imagine a configuration as shown in Figure 1, where electrons move vertically downward to the denser solar atmosphere. A motion tangential to the solar surface would also lead to a limb brightening (Canfield et al., 1986; Dermer, 1987). Petrosian (1985) taking into account the transport of the electrons in the solar atmosphere concluded, that a downward isotropic distribution can explain the concentration of events towards the limb and that mirroring of the electrons is unimportant, if the magnetic fields do not depend strongly on altitude. On the other hand Kocharov et al. (1987, 1988) and Ramaty et al. (1988) assuming a more realistic magnetic field geometry, have shown that electrons injected isotropically at the top of a magnetic loop loose their energy preferentially when they mirror in the converging magnetic field. In their model a beaming of the primary particles along the magnetic field is not a necessary condition to explain the observational fact that flares with photon emission above 
$10 \mathrm{MeV}$ are concentrated towards the limb. Thus, the implication of the limb brightening on the directionality of the primary particles depend to a large degree on the geometry of the magnetic field, which may differ from flare to flare (MacKinnon and Brown, 1989). It was, therefore, proposed to observe flares stereoscopically in the energy bands $0.28-1 \mathrm{MeV}$ and $>10 \mathrm{MeV}$ from different directions with identical detectors (Canfield et al., 1986; Vestrand and Ghosh, 1987). The optimal orbital configuration for stereoscopic observations is obtained if one spacecraft is in an earth orbit and the other in an ecliptic orbit $90^{\circ}$ away in heliocentric longitude.

Information about the angular distribution of the protons cannot be obtained from the observation of the 4-7 MeV fluence, because this emission is only weakly dependent on the proton anisotropy (Ramaty, 1986). The flux of the escaping neutrons, however, depends on the angular distribution of the primary particles. It was shown by Hua and Lingenfelter $(1987 \mathrm{c})$ that the neutron flux observed after the flares of $21 \mathrm{June}, 1980$ and 3 June, 1982, when normalized to the 4-7 MeV emission is consistent with a fan beam or an isotropic distribution of the protons whereas a downward beamed distribution seems to be ruled out from the observations.

It must be noted, however, that this conclusion is based upon the measurements of only two flares. More observations are needed, to determine the angular distribution of the interacting particles.

\section{Conclusion}

The view about the acceleration of particles during solar flares has changed considerably since the early 1980 's, when the Sun was monitored in the gamma-ray regime by the SMM and Hinotori detectors. This was to be expected, because in an environment, where in situ measurements are precluded and the motion of charged particles is constrained by strong and complex magnetic fields, the measurement of neutral radiations provides the closest approach to the flare phenomenon.

The good correlation between the continuum- and gamma-ray line emission, independent of the flare duration and flare size, may be a hint that the primary acceleration of particles proceeds under rather similar conditions from flare to flare.

Evidence for a two-phase acceleration mechanism is obtained by the observation of a distinct temporal development of the high-energy gamma-ray emission during two very intense solar flares. Whereas the second phase may be explained by a shock wave moving through the corona, a candidate which accelerates the particles during the first phase is not yet firmly established. The rapid acceleration to very high energies in one step and the abrupt switching off, inferred from certain gamma-ray measurements (Forrest and Chupp, 1983; Kane et al., 1986) are challenges for any theorist dealing with acceleration mechanisms. In this respect the capabilities of stochastic processes may be strained. Therefore, acceleration by DC-electric fields, which is known to operate in the Earth's magnetosphere, has been investigated as an alternative.

The observation that flares emitting high-energy gamma-rays are concentrated towards the solar limb, suggests that the radiating electrons are anisotropic. It is still a 
matter of debate whether this detection can help to choose between different acceleration mechanisms.

\section{Acknowledgements}

The major contributors to the SMM GRS solar flare data analysis are E. L. Chupp, D. J. Forrest, G. Kanbach, C. Reppin, G. H. Share, and W. T. Vestrand, to whom the author is indebted. This work was partly supported by the Bundesministerium für Forschung und Technologie under 010K017-ZA/WS/WRK0275:4 in West Germany.

\section{References}

Anzer, U. and Tandberg-Hanssen, E.: 1970, Solar Phys. 11, 61.

Bai, T.: 1977, 'Studies of Solar Hard X-Rays and Gamma Rays: Compton Backscatter Anisotropy, Polarization and Evidence for Two Phases of Aceleration', Ph.D. thesis, University of Maryland.

Bai, T.: 1982, in L. E. Lingenfelter, H. S. Hudson, and D. M. Worrall (eds.), AIP, New York, p. 409.

Bai, T.: 1986, Astrophys. J. 308, 912.

Bai, T.: 1988, Astrophys. J. 334, 1049.

Bai, T. and Dennis, B. R.: 1985, Astrophys. J. 292,699.

Bai, T. and Ramaty, R.: 1978, Astrophys. J. 219, 705.

Bai, T. and Ramaty, R.: 1979, Astrophys. J. 227, 1072.

Bai, T., Dennis, B. R., Kiplinger, A. L., Orwig, L. E., and Frost, K. J.: 1983a, Solar Phys. 86, 409.

Bai, T. Hudson, H. S., Pelling, R. M., Lin, R. P., Schwartz, R. A., and von Rosenvinge, T. T.: 1983b, Astrophys. J. 267, 433.

Bespalov, P. A., Zaitsev, V. V., and Stepanov, A. V.: 1987, Solar Phys. 114, 127.

Biermann, L., Haxel, O., and Schlüter, A.: 1951, Z. Naturforsch. 6a, 47.

Blandford, R. P. and Ostriker, J. P.: 1978, Astrophys. J. 221, L29.

Bogovalov, S. V., Kel'ner, S. R., Kotov, Yu. D., Zenchenko, V. M., Vedrenne, G., Barat, C., Chambon, G., and Talon, R.: 1985, Soviet Astron. Letters 11, 322.

Cane, H. V., McGuire, R. E., and von Rosenvinge, T. T.: 1986, Astrophys. J. 301, 448.

Canfield, R. C. et al: 1986, Energetic Phenomena on the Sun, NASA CP-2439, p. 3-1.

Cargill, P. and Vlahos, L.: 1989, in B. M. Haisch and M. Rodonò (eds.), IAU Colloq. 104, Solar and Stellar

Flares, Poster Papers, Publ. Catania Astrophys. Obs., Special Volume, p. 325.

Carlquist, P.: 1986, IEEE Trans. Plasma Sci. PS-14, 794.

Chambon, G., Hurley, K., Niel, M., Talon, R., and Vedrenne, G.: 1981, Solar Phys. 69, 147.

Chupp, E. L.: 1984, Ann. Rev. Aston. Astrophys. 22, 359.

Chupp, E. L.: 1987, Phys. Scripta T18, 5.

Chupp, E. L., Forrest, D. J., Ryan, J. M., Heslin, J., Reppin, C., Pinkau, K., Kanbach, G., Rieger, E., and Share, G. H.: 1982, Astrophys. J. 263, L95.

Chupp, E. L., Forrest, D. J., Vestrand, W. T., Debrunner, H., Flückiger, E., Cooper, J. F., Kanbach, G., Reppin, C., and Share, G. H.: 1985, 19th Int. Cosmic Ray Conf. Papers 4, 126.

Chupp, E. L., Debrunner, H., Flückiger, E., Forrest, D. J., Golliez, F., Kanbach, G., Vestrand, W. T., Cooper, J., and Share, G. H.: 1987, Astrophys. J. 318, 913.

Cliver, E. W., Forrest, D. J., McGuire, R. E., von Rosenvinge, T. T., Reames, D. V., Cane, H. V., and Kane, S. R.: 1987, 20th Int. Cosmic Ray Conf. Papers 3, 61.

Colgate, S. A.: 1978, Astrophys. J. 221, 1068.

Crannell, C. J., Crannell, H., and Ramaty, R.: 1979, Astrophys. J. 229, 762.

Datlowe, D. W., O’Dell, S. L., Peterson, L. E., and Elcan, M. J.: 1977, Astrophys. J. 212, 561.

Debrunner, H., Flückiger, E., Chupp, E. L., and Forrest, D. J.: 1983, 18th Int. Cosmic Ray Conf. Papers 4, 75 .

Decker, R. B. and Vlahos, L.: 1986, Astrophys. J. 306, 710.

Dermer, C. D.: 1987, Astrophys. J. 323, 795. 
Dröge, W. and Schlickeiser, R.: 1986, Astrophys. J. 305, 909.

Dröge, W., Meyer, P., Evenson, P., and Moses, D.: 1989, Solar Phys. 121, 95 (this issue).

Ellison, D. C.: 1984, J. Geophys. Res. 90, 29.

Ellison, D. C. and Ramaty, R.: 1985, Astrophys. J. 298, 400.

Elwert, G. and Haug, E.: 1971, Solar Phys. 20, 413.

Evenson, P., Meyer, P., and Pyle, K. R.: 1983, Astrophys. J. 274, 875.

Evenson, P., Kroeger, R., and Meyer, P.: 1985, 19th Int. Cosmic Ray Conf. Papers 4, 130.

Evenson, P., Meyer, P., Yanagita, S., and Forrest, D. J.: 1984, Astrophys. J. 283, 439.

Evenson, P., Hovestadt, D., Meyer, P., and Moses, D.: 1985, 19th Int. Cosmic Ray Conf. Papers $4,74$.

Forman, M. A.: 1981, Adv. Space Res. 1, 41.

Forman, M. A., Ramaty, R., and Zweibel, E. G.: 1986, in P. A. Sturrock, T. E. Holzer, D. Mihalas, and R. K. Ulrich (eds.), The Physics of the Sun, Vol. II, Chap. 13, D. Reidel Publ. Co., Dordrecht, Holland, p. 249.

Forrest, D. J.: 1983, in M. L. Burns, A. K. Harding, and R. Ramaty (eds.), Positron-Electron Pairs in Astrophysics, AIP, New York, p. 3.

Forrest, D. J. and Chupp, E. L.: 1983, Nature 305, 291.

Forrest, D. J., Vestrand, W. T., Chupp, E. L., Rieger, E., Cooper, J. F., and Share, G. H.: 1985, 19th Int. Cosmic Ray Conf. Papers 4, 146.

Forrest, D. J., Vestrand, W. T., Chupp, E. L., Rieger, E., Cooper, J. F., and Share, G. H.: 1986, Adv. Space Res. 6, No. 6, 115.

Haerendel, G.: 1987, ESA SP-275, 205.

Haerendel, G., Rieger, E., Valenzuela, A., Föppl, H., Stenbaek-Nielsen, H. C., and Wescott, E. M.: 1976, ESA SP-115, 203.

Haug, E.: 1975, Z. Naturforsch. 30a, 1099.

Hua, X.-M. and Lingenfelter, R. E.: 1987a, Solar Phys. 107, 351

Hua, X.-M. and Lingenfelter, R. E.: 1987b, Solar Phys. 113, 229

Hua, X.-M. and Lingenfelter, R. E.: 1987c, Astrophys. J. 323, 779.

Ibragimov, J. A. and Kocharov, G. E.: 1977, Soviet Astron. Letters 3, 211.

Kallenrode, M.-B., Rieger, E., Wibberenz, G., and Forrest, D. J.: 1987, 20th Int. Cosmic Ray Conf. Papers 3,70 .

Kanbach, G., Pinkau, K., Reppin, C., Rieger, E., Chupp, E. L., Forrest, D. J., Ryan, J. M., Share, G. H., and Kinzer, R. L.: 1981, 17th Int. Cosmic Ray Conf. Papers 10, 9.

Kane, S. R.: 1974, in G. Newkirk Jr. (ed.), 'Coronal Disturbances', IAU Symp. 57, 105.

Kane, S. R., Chupp, E. L., Forrest, D. J., Share, G. H., and Rieger, E.: 1986, Astrophys. J. 300, L95.

Kane, S. R., Fenimore, E. E., Klebesadel, R. W., and Laros, J. G.: 1988, Astrophys. J. 326, 1017.

Koch, H. W. and Motz, J. W.: 1959, Rev. Mod. Phys. 31, 920.

Kocharov, G. E.: 1983, Invited Talks 18th Eur. Cosmic Ray Symp., Bologna, p. 51.

Kocharov, G. E.: 1987, in R. A. Syunyaev (ed.), Soviet Sci. Rev. Section E. Astrophys. Space Phys. Vol. 6, Harwood Acad. Publ. G.m.b.H., U.K., p. 155.

Kocharov, G. E., Kovaltsov, G. A., Mandzhavidze, N. Z., and Semukhin, P. E.: 1987, 20th Int. Cosmic Ray Conf. Papers 3, 74.

Kocharov, G. E., Kocharov, L. G., Kovaltsov, G. A., and Mandzhavidze, N. Z.: 1988, Preprint 1258, Academy of Sciences of the USSR, A. F. Ioffe Physico-Technical Institute, Leningrad.

Kozlovski, B., Lingenfelter, R. E., and Ramaty, R.: 1987, Astrophys. J. 316, 801.

MacKinnon, A. L. and Brown, J. C.: 1989, unpublished contribution to IAU Colloq. 104.

MacKinnon, A. L., Brown, J. C., Trottet, G., and Vilmer, N.: 1983, Astron. Astrophys. 119, 297.

McDonald, F. G. and van Hollebeke, M. A. I.: 1985, Astrophys. J. 290, L67.

McGuire, R. E. and von Rosenvinge, T. T.: 1984, Adv. Space Res. 4, Nos. 2-3, 117.

Melrose, D. B. and Brown, J. C.: 1976, Monthly Notices Roy. Astron. Soc. 176, 15.

Miller, J. A. and Ramaty, R.: 1987, Solar Phys. 113, 195.

Miller, J. A., Ramaty, R., and Murphy, R. J.: 1987, 20th Int. Cosmic Ray Conf. Papers 3, 33.

Mozer, F. S., Cattell, C. A., Hudson, M. K., Lysak, R. L., Temerin, M., and Torbert, R. B.: 1980, Space Sci. Rev. 27, 155.

Murphy, R. J. and Ramaty, R.: 1984, Adv. Space Res. 4, No. 7, 127.

Murphy, R. J., Dermer, C. D., and Ramaty, R.: 1987, Astrophys. J. Suppl. 63, 721.

Neustock, H.-H.. Wibberenz, G., and Iwers, B.: 1985, 19th Int. Cosmic Ray Conf. Papers 4, 102. 
Ohsawa, Y. and Sakai, J.-I.: 1987, Astrophys. J. 313, 440.

Pallavicini, R., Serio, S., and Vaiana, G. S.: 1977, Astrophys. J. 216, 108.

Papadopoulos, K., Goodrich, C. C., Cargill, P., and Vlahos, L.: 1985, EOS Trans. Am. Geophys. Union 66, 331.

Pesses, M. E., Klecker, B., Glöckler, G., and Hovestadt, D.: 1981, 17th Int. Cosmic Ray Conf. Papers 3, 36. Petrosian, V.: 1985, Astrophys. J. 299, 987.

Prince, T. A., Forrest, D. J., Chupp, E. L., Kanbach, G., and Share, G. H.: 1983, 18th Int. Cosmic Ray Conf. Papers 4, 79.

Ramaty, R.: 1979, in J. Aarons, C. Max, and C. McKee (eds.), Particle Acceleration Mechanisms in Astrophysics, AIP, New York, p. 135

Ramaty, R.: 1986, in P. A. Sturrock, T. E. Holzer, D. Mihalas, and R. K. Ulrich (eds.), The Physics of the Sun, Vol. II, Cap. 14, D. Reidel Publ. Co., Dordrecht, Holland, p. 291.

Ramaty, R. and Murphy, R. J.: 1987, Space Sci. Rev. 45, 213.

Ramaty, R., Kozlovsky, B., and Lingenfelter, R. E.: 1975, Space Sci. Rev. 18, 341.

Ramaty, R., Kozlovsky, B., and Suri, A. N.: 1977, Astrophys. J. 214, 617.

Ramaty, R., Kozlovsky, B., and Lingenfelter, R. F.: 1979, Astrophys. J. Suppl. 40, 487

Ramaty, R., Murphy R. J., and Dermer, C. D.: 1987, Astrophys. J. 316, L41.

Ramaty, R., Murphy, R. J., Kozlovsky, B., and Lingenfelter, R. E.: 1983, Solar Phys. 86, 395.

Ramaty, R., Miller, J. A., Hua, X.-M., and Lingenfelter, R. E.: 1988, in G. H. Share and N. Gehrels (eds.), Nuclear Spectroscopy of Astrophysical Sources, AIP, New York (in press).

Rieger, E.: 1982, in Hinotori Symp. on Solar Flares (Tokyo Inst. Space Astronautical Sci.), p. 246.

Rieger, E., Reppin, C., Kanbach, G., Forrest, D. J., Chupp, E. L., and Share, G. H.: 1983, 18th Int. Cosmic Ray Conf. Papers 10, 338.

Rieger, E., Forrest, D. J., Bazilevskaya, G., Chupp, E. L., Kanbach, G., Reppin, C., and Share, G. H.: 1987, 20th Int. Cosmic Ray Conf. Papers 3, 65.

Ryan, J. M.: 1986, Solar Phys. 105, 365.

Sakai, J.-I. and Ohsawa, Y.: 1987, Space Sci. Rev. 46, 113.

Scholer, M.: 1988, in O. Havnes et al. (eds.), Activity in Cool Star Envelopes, Kluwer Academic Publishers, Dordrecht, Holland, p. 195.

Share, G. H., Chupp, E. L., Forrest, D. J., and Rieger, E.: 1983, in M. L. Burns, A. K. Harding, and R. Ramaty (eds.), Positron-Electron Pairs in Astrophysics, AIP, New York, p. 15.

Spicer, D. S.: 1982, Space Sci. Rev. 31, 351.

Sturrock, P.: 1989, Solar Phys. 121, 387 (this issue).

Temerin, R. B., Cattell, C. A., Mozer, F. S., and Meng, C.-I.: 1982, Phys. Rev. Letters 48, 1175.

van Hollebeke, M. A. I.: 1979, Rev. Geophys. Space Phys. 17, 545.

van Hollebeke, M. A. I., Ma Sung, L. S., and McDonald, F. B.: 1975, Solar Phys. 41, 189.

Vestrand, W. T. and Ghosgh, A.: 1987, 20th Int. Cosmic Ray Conf. Papers 3, 57.

Vestrand, W. T., Forrest, D. J., Chupp, E. L., Rieger, E., and Share, G. H.: 1987, Astrophys. J. 322, 1010 Vilmer, N., Kane, S. R., and Trottet, G.: 1982, Astron. Astrophys. 108, 306.

Vlahos, L. et al:: 1986, Energetic Phenomena on the Sun, NASA, CP-2439, p. 2-1.

von Rosenvinge, T. T., Ramaty, R., and Reames, D. V.: 1981, 17th Int. Cosmic Ray Conf. Papers 3, 28.

Wang, H. T. and Ramaty, R.: 1974, Solar Phys. 36, 129.

Wescott, E. M., Stenbaek-Nielsen, H. C., Hallinan, T. J., Davis, T. N., and Peek, H. M.: 1976, J. Geophys. Res. 81, 4495 .

Wild, J. P., Smerd, S. F., and Weiss, A. A.: 1963, Ann. Rev. Astron. Astrophys. 1, 291.

Yang, J., Turner, M. S., Steigman, G., Schramm, D. N., and Olive, K. A.: 1982, Astrophys. J. 281, 493

Yoshimori, M. and Watanabe, H.: 1985, 19th Int. Cosmic Ray Conf. Papers 4, 90.

Yoshimori, M., Okudaira, K., Hirasima, Y., and Kondo, I.: 1983, Solar Phys. 86, 375. 